\title{
Utilidad de los antagonistas de los receptores muscarínicos en el tratamiento de los varones con sintomas del tracto urinario inferior secundarios a HBP
}

\author{
Steven A, Kaplan MD. \\ Departament of Urology. Institute of Bladder and Prostate Health. Weill Cornell Medical College. New York.
}

Actas Urol Esp. 2007;31(2):86-91

\section{RESUMEN}

UTILIDAD DE LOS ANTAGONISTAS DE LOS RECEPTORES MUSCARÍNICOS EN EL TRATAMIENTO DE LOS VARONES CON SÍNTOMAS DEL TRACTO URINARIO INFERIOR SECUNDARIOS A HBP

El uso de antimuscarínicos en pacientes varones con síntomas del tracto urinario inferior (STUI) por hiperplasia benigna de próstata (HBP) con síntomas de vejiga hiperactiva (VH) es un tema que está adquiriendo relevancia en los últimos años. Indicar estas drogas choca directamente contra un concepto estudiado en la facultad: los antimuscarinicos están contraindicados en pacientes con HBP. Sin embargo, diversos factores han conducido a que este tema esté en constante revisión. La creación del síndrome de VH y toda la investigación científica llevada a cabo durante los últimos años han aportado datos epidemiológicos y fisiopatológicos que merecen la pena repasar. Sobre todo, para poder entender el porqué se ha planteado la revisión de este concepto solidamente establecido desde hace muchos años. En este trabajo se realiza una revisión de la prevalencia de la VH en varones con HBP, del papel de la urodinámica en la evaluación de varones con VH y de la evidencia existente sobre el efecto que los antimuscarínicos tienen en cuanto a eficacia y seguridad en los pacientes varones con obstrucción e hiperactividad del detrusor.

Palabras clave: Vejiga hiperactiva en hombres. Antimuscarinicos.

\section{ABSTRACT \\ THE ROLE OF MUSCARINIC RECEPTOR ANTAGONISTS IN THE TREATMENT OF MEN WITH LOWER URINARY TRACT SYMPTOMS SECONDARY TO BPH.}

Use of antimuscarinic agents in male patients with lower urinary tract symptoms (LUTS) secondary to benign prostatic hyperplasia (BPH) and with symptoms of overactive bladder (OAB) has become increasingly relevant over the last few years. Prescription of these drugs is in conflict with a long-established concept learnt in Medical School: antimuscarinics are contraindicated in patients with BPH. Several factors, however, have placed this issue under constant review. The description of OAB syndrome and the scientific research carried out over recent years have provided epidemiological and pathophysiological data worthwhile reviewing, most importantly in order to understand why a review of such a solidly established concept has been raised. This paper reviews the prevalence of OAB in men with $\mathrm{BPH}$, the role of urodynamics to evaluate men with $\mathrm{OAB}$ and the existing evidence on the effect antimuscarinics have in terms of efficacy and safety on male patients with detrusor obstruction and overactivity.

Keywords: Male overactive bladder. Antimuscarinics. 
$\mathrm{E}_{\mathrm{p}}^{1}$ modelo de tratamiento médico de la hiperplasia benigna de próstata (HBP) ha sufrido un cambio radical durante el último decenio. Guiados por las nuevas investigaciones, la aprobación de nuevos fármacos y tratamientos mínimamente invasivos y la economía de la financiación sanitaria, los médicos han modificado su método de atención al paciente en distintas formas. Esta evolución del pensamiento continúa en el momento actual.

La polaquiuria y la nicturia son síntomas del tracto urinario inferior (STUI) que se observan secundariamente a hiperplasia benigna de próstata (HBP), obstrucción infravesical (OIV) y vejiga hiperactiva (VHA): estos síntomas son muy frecuentes y su prevalencia en el varón aumenta con la edad. Con independencia de su etiología, el objetivo del tratamiento de los síntomas sería mejorar la calidad de vida y evitar la progresión clínica. La duda surge cuando un varón con síntomas de VHA tiene HBP o OIV; el riesgo de retención urinaria ¿es un factor que contraindica la utilización de antagonistas de los receptores muscarínicos? El uso de estos antagonistas en varones con STUI secundarios a HBP es un tema en evolución. El propósito de esta revisión es analizar la compleja relación entre VHA/STUI/ HBP/OIV, la utilidad diagnóstica de la urodinámica y el efecto terapéutico beneficioso de los fármacos.

\section{Prevalencia e importancia de la VHA en el varón}

La VHA es un complejo sintomático definido específicamente como "necesidad imperiosa de orinar, con o sin incontinencia de urgencia, habitualmente con polaquiuria y nicturia... si no existe una infección demostrada u otra afección obvia" ${ }^{1}$. La vejiga hiperactiva (VHA) es un síndrome caracterizado por necesidad imperiosa de orinar, con o sin incontinencia urinaria de urgencia (IUU), habitualmente con polaquiuria y nicturia ${ }^{1}$. Los síntomas de la VHA son casi idénticos a los sintomas de STUI llenado del tracto urinario inferior, y se calcula que afectan al 16\% de los varones y al $17 \%$ de las mujeres en EEUU $^{2,3}$. En otro estudio, Milsom et al. demostraron que la prevalencia de sintomas de VHA aumenta con la edad: $42 \%$ de los varones y $31 \%$ de las mujeres mayores de 75 años $^{4}$. Los sintomas de VHA tienen efectos adversos en la calidad de vida (CdV), con aumento de los sintomas depresivos y disminución de la calidad del sue$\tilde{n}^{3}$. El National Overactive Bladder Evaluation program reveló que la prevalencia global de VHA en varones era del 16\% y también aumentaba con la edad ${ }^{5}$.

Los síntomas de STUI en el varón obedecen a múltiples causas, entre las que se encuentran HBP, disfunción primaria del cuello de la vejiga, estenosis uretral y disfunción neurológica. Los síntomas de VHA a menudo están causados por hiperactividad del detrusor (HD), un diagnóstico urodinámico caracterizado por contracciones involuntarias durante la fase de llenado de la vejiga $^{6,7}$. El tratamiento de la HD y de los síntomas de VHA resultantes puede presentar problemas particulares en el varón, debido a la significativa comorbilidad HBP.

La HBP puede producir OIV, que a menudo coexiste con HD; la OIV puede producir HD por isquemia o denervación del músculo detrusor ${ }^{8-10}$. La OIV a menudo se caracteriza y se acompaña por aumento del contenido de colágeno del detrusor $^{11}$. Todos estos trastornos pueden originar problemas de vaciamiento, de llenado o de ambos tipos, causantes de sintomas miccionales tanto irritativos como obstructivos. En los varones con STUI moderados o intensos existe un mayor riesgo de retención urinaria o de progresión de los sintomas ${ }^{12}$

\section{Utilidad de la urodinámica en la evaluación de la VHA en el varón}

Numerosos datos urodinámicos indican la coexistencia de HD y OIV. Revisamos los registros urodinámicos de más de 2.800 varones consecutivos mayores de 50 años a los que se evaluó por STUI. La OIV estaba presente en el $62 \%$ de los varones y, de ellos, el 66\% tenían HD concomitante $^{13}$. Esto concuerda con otras series que refieren OIV y HD concomitantes en el 40\%-60\% de los varones ${ }^{14,17}$.

Para determinar la utilidad de la urodinámica en el diagnóstico y tratamiento de la VHA es preciso tener en cuenta la compleja relación existente entre los sintomas de VHA y las mediciones urodinámicas. Es habitual decir que la hiperacti- 
vidad del detrusor causa síntomas de VHA. Sin embargo, la relación directa entre los síntomas de VHA y los datos urodinámicos ha sido difícil de establecer ${ }^{18,19}$. Las puntuaciones de síntomas (p. ej., con I-PSS) no siempre son predictivas de hallazgos urodinámicos ${ }^{20-22}$.

Los síntomas específicos de VHA, la necesidad imperiosa de orinar y la incontinencia de urgencia, tienen una relación más estrecha con el diagnóstico urodinámico de HD. En un estudio de 160 varones con STUI sometidos a evaluación urodinámica, el 68\% tenía OIV y el 46\% HD concomitante. La incontinencia de urgencia guardó relación con la presencia de $\mathrm{HD}^{23}$. En otro estudio, la presencia de HD tuvo relación con la percepción del sintoma de necesidad imperiosa de orinar y con la calidad de vida en el I-PSS ${ }^{24}$. En definitiva, todavía está por determinar el valor diagnóstico en la evaluación de los síntomas de vías urinarias bajas y, más específicamente, de la VHA en varones.

\section{Efectos de los antagonistas de los receptores muscarínicos en varones con HD y OIV}

Es verosímil creer que los varones que presentan síntomas de VHA sin indicios urodinámicos concomitantes de OIV tienen menos probabilidad de responder a los tratamientos dirigidos a la próstata (p. ej., antagonistas del receptor alfa, inhibidores de la 5-alfa-reductasa). Los antagonistas uroselectivos de los receptores adrenérgicos alfa $_{1}$ (p. ej., tamsulosina, alfuzosina) a menudo se eligen como tratamientos iniciales en la HBP sintomática. Sin embargo, la escasa densidad de receptores alfa en el detrusor puede imposibilitar los efectos directos de los alfabloqueantes sobre la contractibilidad del detrusor, y estos fármacos han demostrado poca eficacia en el tratamiento de los síntomas de VHA ${ }^{25,26}$. Los inhibidores de la 5-alfa-reductasa (p. ej., finasterida, dutasterida) inhiben la conversión de testosterona en el más potente andrógeno 5-alfa-dihidrotestosterona (DHT) y también se utilizan para tratar los síntomas de HBP. Sin embargo, es poco probable que las modificaciones endocrinas mediadas por los inhibidores de la 5-alfa-reductasa atenúen la HD y los síntomas debidos a VHA. Está claro que se necesita una nueva eva- luación de la eficacia y la seguridad de los fármacos de la VHA en varones con o sin OIV, y que se deben desarrollar estrategias para el uso adecuado de estos fármacos.

El fundamento de la utilización de antagonistas de los receptores muscarinicos para aliviar los sintomas de VHA está bien demostrado. En parte, se basa en la premisa de que la contractilidad del detrusor está regulada por el sistema nervioso parasimpático a través de vías colinérgicas muscarínicas. En consecuencia, estos fármacos están reconocidos como tratamiento de primera línea de los síntomas de VHA, con seguridad y eficacia demostradas ${ }^{27}$.

Dado que los antagonistas de los receptores muscarínicos mejoran los síntomas de VHA, ¿por qué no se han empleado en varones con la misma frecuencia que en mujeres? De hecho, la enseñanza urológica tradicional dice que estos fármacos no se deben utilizar en varones con síntomas de VHA e HBP, por el temor a disminuir la contractilidad de la vejiga y agravar los síntomas obstructivos, con la posibilidad de causar una retención urinaria aguda. Pero, ¿hay pruebas de que esto sea válido? De hecho, datos más recientes indican que estos fármacos son seguros en este grupo de varones.

En un estudio multicéntrico, multinacional y doble ciego se examinaron los problemas de seguridad derivados de tratar con tolterodina de liberación inmediata a varones con OIV y HD diagnosticadas urodinámicamente ${ }^{28}$. En dicho estudio se aleatorizó a 221 varones para recibir tolterodina $2 \mathrm{mg}$ dos veces al día $(\mathrm{n}=149)$ o placebo $(n=72)$. Durante tres meses se controló a estos pacientes con estudios urodinámicos y en busca de acontecimientos adversos. Se excluyó a los pacientes con un volumen de orina residual posmiccional $\geq 40 \%$ de la capacidad cistométrica máxima y a los intervenidos previamente de próstata o vejiga. La mayoría de los pacientes tenían OIV moderada o intensa, y se les distribuyó de forma uniforme en los grupos de tolterodina y placebo. Los resultados indican que no hubo diferencias significativas entre tolterodina y placebo en lo que respecta a la incidencia de retención urinaria aguda (un paciente en cada grupo) o de abandono del estudio por acontecimientos adversos $(6,0 \%$ con tolterodina y $6,9 \%$ con placebo). 
Las variaciones del índice de flujo urinario máximo (Q máx) y de la presión del detrusor al flujo máximo (Pdet Qmáx) con respecto al momento basal en los receptores de tolterodina fueron estadísticamente equivalentes a las observadas con placebo. Hay que destacar que la mediana del aumento del residuo posmiccional (RPM) fue significativamente mayor, en términos estadísticos, en el grupo de tolteradina que en el grupo placebo; tal aumento fue clínicamente significativo. Una pregunta obvia es ¿qué les habría sucedido a los pacientes con RPM más altas en el momento basal que fueron excluidos del estudio? ¿Presentan mayor riesgo de retención urinaria con la medicación anticolinérgica?

En otro estudio se examinó el uso de la combinación de tolterodina y tamsulosina (un alfabloqueante) en varones con OIV y HD concomitante ${ }^{29}$. Se evaluó a 50 varones griegos consecutivos. Todos los pacientes recibieron tamsulosina $0,4 \mathrm{mg}$ al día; se eligió aleatoriamente a 25 (50\%) de ellos para tomar también tolterodina 2 mg dos veces al día. Se obtuvieron puntuaciones de la calidad de vida (Urolife ${ }^{T M} \mathrm{QOL}$ ) y evaluaciones urodinámicas en el momento basal y a los tres meses de tratamiento. Dos pacientes (8\%) del grupo de tolterodina abandonaron el estudio por sequedad de boca, mientras que un paciente de cada grupo abandonó por hipotensión ortostática atribuida a tamsulosina. Sólo los pacientes que recibieron el tratamiento combinado (tamsulosina y tolterodina) experimentaron mejorías estadísticamente significativas de las puntuaciones de CdV. Los varones tratados con una combinación de un alfabloqueante y un antagonista de los receptores muscarínicos experimentaron una disminución significativa de la presión máxima del detrusor durante la micción, un aumento significativo de la capacidad de la vejiga, una menor presión de contracción inestable máxima y un mayor volumen en la primera contracción inestable. Ambos grupos presentaron un aumento estadísticamente significativo del flujo y el volumen máximos en la primera contracción inestable. Y, lo más importante, ningún paciente sufrió retención urinaria aguda durante el estudio, entre cuyas limitaciones se encontraban la falta de un grupo controlado con placebo y la corta duración del tratamiento.
Un estudio más reciente es el publicado por Kaplan et al. ${ }^{30}$. En un ensayo prospectivo, estos autores evaluaron la seguridad y la eficacia de tolterodina de liberación retardada en varones con STUI que no habian respondido al tratamiento alfabloqueante previo. En este estudio, 43 varones consecutivos con síntomas de vías urinarias bajas secundarios a HBP y que habian sido tratados sin éxito con alfabloqueantes comenzaron con tolterodina de liberación retardada 4 mg una vez al día en monoterapia durante 6 meses.

Completaron los 6 meses del estudio 39 (91\%) de los 43 pacientes incluidos. El promedio de edad de los pacientes era de 61 años (límites, 50-83 años) y la duración media del tratamiento previo con alfabloqueantes fue de 5,7 meses. Los alfabloqueantes utilizados fueron tamsulosina $(n=30)$, doxazosina $(n=9)$ y terazosina $(n=4)$, y el tratamiento fracasó por efectos adversos $(n=11)$ y falta de eficacia ( $n=32$ ). La cifra media basal de PSA era de 2,3 $\mathrm{ng} / \mathrm{ml}$ y la puntuación media basal del dominio de función eréctil de IIEF, de $32,1 \pm 9,8$.

La frecuencia urinaria descendió de 9,8 a 6,3 micciones al día $(P<0,03)$ y los episodios de nicturia disminuyeron desde 4,1 hasta 2,9 por noche $(P<0,01)$. Las variaciones de la media de las puntuaciones de sintomas AUA $(-6,1$, $P<0,001), Q_{\max }(+1,9 \mathrm{ml} / \mathrm{s}, P<0,001)$ y volúmenes RPM $(-22 \mathrm{ml}, P<0,03)$ después de 6 meses de tratamiento con tolterodina ER fueron estadísticamente significativas (Tabla 1). Es importante señalar que las puntuaciones totales de síntomas AUA disminuyeron significativamente $(-6,0)$ después de sólo 1 mes de tratamiento $(P<0,02)$. Las puntuaciones medias de todos los sintomas individuales de VHA y de vaciamiento descendieron significativamente después de 6 meses de tratamiento con tolterodina ER (valores $P<0,02$; Tabla 2).

Se observó una función eréctil normal en 27 varones (63\%) en el momento basal y en 29 (67\%) después de 6 meses de tratamiento. La media de las puntuaciones totales del dominio de función eréctil del IIEF aumentó desde 12,7 $\pm 4,3$ en el momento basal hasta 19,6 $\pm 5,7$ después de 6 meses de tratamiento con tolterodina ER. No hubo variaciones de la función eyaculatoria. Cuatro varones (9\%) suspendieron el tratamiento 
Tabla 1

Volumen RPM con el tratamiento con tolterodina ER

\begin{tabular}{lcccc}
\hline & n & $\begin{array}{c}\text { Puntuación AUA } \\
\text { Media } \pm \mathbf{~ D E ~}\end{array}$ & $\begin{array}{c}\mathbf{Q}_{\max }(\mathbf{m l} / \mathbf{s}) \\
\text { RPM (ml) }\end{array}$ & \\
\hline Basal & 43 & $17,3 \pm 3,2$ & $9,8 \pm 3,8$ & $97 \pm 3,4$ \\
1 mes & 43 & $11,3 \pm 2,7$ & $11,4 \pm 2,2$ & $79 \pm 2,7$ \\
3 meses & 39 & $10,7 \pm 3,1$ & $10,9 \pm 2,1$ & $72 \pm 3,1$ \\
6 meses & 39 & $11,2 \pm 2,8$ & $11,7 \pm 2,9$ & $75 \pm 3,6$ \\
Variación neta & & $-6,0$ & $+1,9$ & $-22,0$ \\
Valor p & & $<0,001$ & $<0,001$ & $<0,03$ \\
\hline
\end{tabular}

AUA = Americal Urological Association; ER=liberación retardada; $\mathrm{RPM}=$ residual posmiccional; $\mathrm{Qmax}=i$ ndice de flujo urinario máximo; $\mathrm{DE}=$ desviación estándar.

con tolterodina ER por sequedad de boca intolerable. No hubo casos de retención urinaria.

El presente estudio era a pequeña escala y no se utilizó un diseño doble ciego controlado con placebo. Además, no se verificó urodinámicamente el estado obstructivo de los pacientes participantes. Sin embargo, los resultados indican que tolterodina ER es un tratamiento eficaz y bien tolerado para los STUI secundarios a HBP, en ausencia o en presencia de OIV. Además, estos datos indican que tolterodina $\mathrm{ER}$ es eficaz en varones que no han respondido al tratamiento con alfabloqueantes.

Por el momento no se han publicado ensayos aleatorizados y controlados que describan los

\section{Tabla 2}

Síntomas de VHA y miccionales con el tratamiento con tolterodina ER

\begin{tabular}{cccc}
\hline Ítem de AUA & Basal & $\mathbf{6}$ meses & Valor p \\
\hline VHA & \multicolumn{2}{c}{ Media \pm DE } & \\
2 & $1,8 \pm 1,1$ & $1,2 \pm 0,6$ & $<0,01$ \\
4 & $2,1 \pm 1,3$ & $1,2 \pm 0,7$ & $<0,001$ \\
7 & $3,6 \pm 2,3$ & $2,4 \pm 1,2$ & $<0,02$ \\
Total & $7,5 \pm 3,1$ & $4,8 \pm 2,1$ & \\
Micción & & & \\
1 & $2,6 \pm 0,9$ & $1,7 \pm 0,7$ & $<0,01$ \\
3 & $2,1 \pm 1,1$ & $1,2 \pm 0,5$ & $<0,001$ \\
5 & $2,7 \pm 0,8$ & $1,8 \pm 0,7$ & $<0,02$ \\
6 & $2,5 \pm 1,1$ & $1,7 \pm 0,9$ & $<0,01$ \\
Total & $9,9 \pm 3,7$ & $6,4 \pm 2,6$ & \\
\hline
\end{tabular}

AUA = American Urological Association; ER= extended release (liberación retardada); VHA = vejiga hiperactiva; $\mathrm{DE}=$ desviación estándar. efectos de otros anticolinérgicos o de formulaciones de liberación retardada de estos fármacos. También serían ideales e informativos estudios prospectivos de formulaciones de liberación retardada en varones con HD y OIV concomitantes.

En conclusión, con la edad aumenta en el varón la prevalencia tanto de VHA como de OIV secundarias al incremento de HBP. Se considere o no que los síntomas de VHA son secundarios a OIV, el objetivo del tratamiento sigue siendo mejorar la calidad de vida al tiempo que se evita el deterioro clínico.

\section{REFERENCIAS}

1. Abrams P, Cardozo L, Fall M, Griffiths D, Rosier P, Ulmsten $\mathrm{U}$, et al. The standardisation of terminology of lower urinary tract function: report from the Standardisation Subcommittee of the International Continence Society. Neurourol Urodyn. 2002;21(2):167-178.

2. Stewart WF, Van Rooyen JB, Cundiff GW, Abrams P, Herzog AR, Corey R, et al. Prevalence and burden of overactive bladder in the United States. World $\mathrm{J}$ Urol. 2003;20(6):327-336.

3. Milsom I, Abrams P, Cardozo L, Roberts RG, Thuroff J, Wein AJ. How widespread are the symptoms of an overactive bladder and how are they managed? A populationbased prevalence study. BJU Int. 2001;87(9):760-766.

4. Girman CJ, Jacobsen SJ, Tsukamoto T, Richard F, Garraway WM, Sagnier PP, et al. Health-related quality of life associated with lower urinary tract symptoms in four countries. Urology. 1998;51(3):428-436.

5. Stewart W, Herzog, AR, Wein A, et al. Prevalence and impact of overactive bladder in the US: results from the NOBLE program. Neurourol Urodyn 2001, 20: 406.

6. Abrams P, Cardozo L, Fall M, Griffiths D, Rosier P, Ulmsten $\mathrm{U}$, et al. The standardisation of terminology in lower urinary tract function: report from the standardisation subcommittee of the International Continence Society. Urology. 2003;61(1):37-49.

7. Fusco F, Groutz A, Blaivas JG, Chaikin DC, Weiss JP. Videourodynamic studies in men with lower urinary tract symptoms: a comparison of community based versus referral urological practices. J Urol. 2001;166(3):910-913.

8. Greenland JE, Brading AF. The effect of bladder outflow obstruction on detrusor blood flow changes during the voiding cycle in conscious pigs. J Urol. 2001;165(1):245-248.

9. Harrison SC, Hunnam GR, Farman P, Ferguson DR, Doyle PT. Bladder instability and denervation in patients with bladder outflow obstruction. Br J Urol. 1987;60(6):519-522.

10. Mirone V, Imbimbo C, Sessa G, Palmieri A, Longo N, Granata AM, et al. Correlation between detrusor collagen content and urinary symptoms in patients with prostatic obstruction. J Urol. 2004;172(4 Pt 1):1386-1389. 
11. Seki N, Karim OM, Mostwin JL. Changes in electrical properties of guinea pig smooth muscle membrane by experimental bladder outflow obstruction. Am J Physiol. 1992; 262(5 Pt 2):885-891.

12. Dmochowski R, Staskin D. Overactive bladder in men: special considerations for evaluation and management. Urology. 2002;60(5 Suppl 1):56-62; discussion 62-63.

13. Kaplan SA, Bowers DL, Te AE, Olsson CA. Differential diagnosis of prostatism: a 12-year retrospective analysis of symptoms, urodynamics, and satisfaction with therapy. J Urol. 1996; 155 (4):1305-1308.

14. Eckhardt MD, van Venrooij GE, Boon TA. Interactions between prostate volume, filling cystometric estimated parameters, and data from pressure-flow studies in 565 men with lower urinary tract symptoms suggestive of benign prostatic hyperplasia. Neurourol Urodyn. 2001;20(5):579590.

15. Eckhardt MD, van Venrooij GE, Boon TA. Symptoms and quality of life versus age, prostate volume, and urodynamic parameters in 565 men with lower urinary tract symptoms suggestive of benign prostatic hyperplasia. Urology. 2001;57(4):695-700.

16. Hyman MJ, Groutz A, Blaivas JG. Detrusor instability in men: Correlation of lower urinary tract symptoms with urodynamic findings. J Urol. 2001;166(2):550-552;discussion 553.

17. Knutson T, Edlund C, Fall M, Dahlstrand C. BPH with coexisting overactive bladder dysfunction an everyday urological dilemma. Neurourol Urodyn. 2001;20(3):237-247.

18. Abdel-Aziz, KF, LeMack G. Overactive bladder in the male patient: bladder, outlet, or both? Current Urology Reports. 2002;3(6):445-451.

19. Hyman MJ, Groutz A, Blaivas JG. Detrusor instability in men: Correlation of lower urinary tract symptoms with urodynamic findings. J Urol. 2001;166(2):550-552;discussion 553.

20. Eckhardt MD, van Venrooij GE, Boon TA. Symptoms and quality of life versus age, prostate volume, and urodynamic parameters in 565 men with lower urinary tract symptoms suggestive of benign prostatic hyperplasia. Urology. 2001; 57(4):695-700.

21. Wadie BS, Ebrahim el-HE, Gomha MA. The relationship of detrusor instability and symptoms with objective parameters used for diagnosing bladder outlet obstruction: a prospective study. J Urol. 2002;168(1):132-134.
22. Hyman MJ, Groutz A, Blaivas JG. Detrusor instability in men: Correlation of lower urinary tract symptoms with urodynamic findings. J Urol. 2001;166:550-552;discussion 553.

23. Wadie BS, Ebrahim el-HE, Gomha MA. The relationship of detrusor instability and symptoms with objective parameters used for diagnosing bladder outlet obstruction: a prospective study. J Urol. 2002;168(1):132-134.

24. Kaplan SA, Te AE, Blaivas JG. Urodynamic findings in patients with diabetic cystopathy. J Urol. 1995;153(2): 342-344.

25. Goepel M, Wittmann A, Rubben H, Michel MC. Comparison of adrenoceptor subtype expression in porcine and human bladder and prostate. Urol Res. 1997;25(3): 199206.

26. Lee JY, Kim HW, Lee SJ, Koh JS, Suh HJ, Chancellor MB. Comparison of doxazosin with or without tolterodine in men with symptomatic bladder outlet obstruction and an overactive bladder. BJU Int. 2004;94(6):817-820.

27. Abrams P, Kaplan SA, De Koning Gans HJ, Millard R. Safety and tolerability of tolterodine for the treatment of overactive bladder in men with bladder outlet obstruction. J Urol. 2006;175(3 Pt 1):999-1004; discussion 1004.

28. Athanasopoulos A, Gyftopoulos K, Giannitsas K, Fisfis J, Perimenis P, Barbalias G. Combination treatment with an alpha-blocker plus an anticholinergic for bladder outlet obstruction: a prospective, randomized, controlled study. J Urol. 2003, 169(6):2253-2256.

29. Dmochowski RR, Staskin DR. Advances in drug delivery: Improved bioavailability and drug effect. Current Urology Reports. 2002;3(6):439-444.

30. Kaplan SA, Walmsley K, Te AE. Use of muscarinic receptor antagonist as monotherapy in men with lower urinary tract symptoms who failed previous therapy with alpha blockers. J Urol. in press.

Correspondencia autor: Dr. A.Steven

Department of Urology. Weill Cornell Medical College

F9 West, Box 261. 1300 York Avenue New York, NY 10021

E-mail autor: kaplans@med.cornell.edu

Información artículo: Original - Revisión

Trabajo recibido: diciembre 2006

Trabajo aceptado: enero 2007 